\title{
Seasonal variation in growth and survival of Strombus canarium (Linnaeus, 1758) larvae.
}

\begin{abstract}
This study was conducted to analyze variation in Strombus canarium larvae development, growth and survivals when cultured during wet (main reproductive period) and dry seasons. Larvae were reared at 200 larvae L(-1) in filtered seawater $(0.22$ microm $)$ and fed with Isochrysis galbana at 1000 cells $\mathrm{mL}(-1)$ ad libitum. The culture environment was maintained at $29+/-1$ degrees C, salinity of $30+/-1$ PSU and photoperiod of 12:12 light dark cycle. Growth of the larvae was described on a length-at-age basis using the modified Gompertz regression. There was high correlation in shell length-at-age relationship for both wet season $(\mathrm{R} 2=0.99)$ and dry season $(\mathrm{R} 2=0.98)$ culture experiments. The maximal growth rate $(\mathrm{M})$ and survival rate $(S)$ were higher for larvae cultured during wet season $(\mathrm{M}=62.44$ microm day(-1), $\mathrm{S}=14.36-2.31 \%)$, compared with dry season $(\mathrm{M}=43.05$ microm day(-1), $\mathrm{S}=5+/-$ $1.15 \%)$. The maximal attainable larval size (a) was however lower during wet season (950.19 +/- 66.93 microm shell length) compared with dry season (1343.05 +/- 586.51 microm shell length), which might be due to significantly low larvae density in the latter. Further studies are needed to investigate variation in bio-chemical composition of the egg mass, which was suggested as the main reason for the differences.
\end{abstract}

Keyword: Mollusk; Veliger; Dog conch; Larval growth rate. 